\title{
Laparoscopic simple prostatectomy vs bipolar plasma enucleation of the prostate in large benign prostatic hyperplasia: a two-center 3-year comparison
}

\author{
Riccardo Lombardo ${ }^{1}$. Anton Zarraonandia Andraca ${ }^{2}$. Cristina Plaza Alonso ${ }^{2}$ - Juan Andres González-Dacal ${ }^{2}$. \\ Higinio Rodríguez Núñez ${ }^{2}$. Aaron Barreiro Mallo ${ }^{2}$ - Barbara Cristina Gentile ${ }^{1} \cdot$ Giorgia Tema ${ }^{1}$. Luca Albanesi ${ }^{1}$. \\ Luca Mavilla ${ }^{1}$. Valeria Baldassarri ${ }^{3,4}\left({ }^{10} \cdot\right.$ Cosimo De Nunzio ${ }^{3} \cdot$ Andrea Tubaro $^{3} \cdot$ Manuel Ruibal Moldes $^{2}$. \\ Roberto Giulianelli ${ }^{1}$
}

Received: 22 May 2020 / Accepted: 24 October 2020 / Published online: 11 November 2020

(c) The Author(s) 2020

\begin{abstract}
Purpose To compare surgery outcomes and safety of button bipolar enucleation of the prostate vs laparoscopic simple prostatectomy in patients with large prostates $(>80 \mathrm{~g})$ in a two-center cohort study.

Methods All patients with lower urinary tract symptoms due to benign prostatic enlargement (Prostate volume $>80 \mathrm{cc}$ ) undergoing button bipolar enucleation of the prostate (BTUEP) or laparoscopic simple prostatectomy (LSP) in two centers were enrolled. Data on clinical history, physical examination, urinary symptoms, uroflowmetry and prostate volume were collected at $0,1,36,12,24$ and 36 months. Early and long-term complications were recorded.

Results Overall, 296 patients were enrolled. Out of them, 167/296 (56\%) performed a LSP and 129/296 (44\%) performed a BTUEP. In terms of efficacy both procedures showed durable results at three years with a reintervention rate of $8 \%$ in the LSP group and of 5\% in the BTUEP group. In terms of safety, BTUEP and LSP presented similar safety profiles with a $9 \%$ of transfusion rate and no major complications.

Conclusion LSP and BTUEP are safe and effective in treating large-volume adenomas with durable results at three years when performed in experienced centers.
\end{abstract}

Keywords BPH $\cdot$ Laparoscopic simple prostatectomy $\cdot$ BTUEP $\cdot$ LUTS $\cdot$ Prostatic disease

\section{Introduction}

Transurethral resection of the prostate (TURP) is considered the standard procedure for men with prostates between 30 and $80 \mathrm{~mL}[1,2]$. In patients with larger prostates $(>80 \mathrm{cc})$, open prostatectomy (OP), holmium laser enucleation (HoLep) and bipolar enucleation (BTUEP) of the prostate represent the gold standard procedures for the management of patients with benign prostatic obstruction (BPO). The management of large adenomas is always challenging and

Riccardo Lombardo and Anton Zarraonandia Andraca contributed equally to the manuscript.

Valeria Baldassarri

v.baldassarri90@gmail.com

Extended author information available on the last page of the article nowadays, the chosen technique often lies on availability and surgeon preference.

According to the latest EAU guidelines, the available techniques (OP; HoLep and bipolar enucleation) in the management of large adenomas $(>80 \mathrm{cc})$ may be considered equal in terms of short- and long-term functional outcomes [2]. The main limitation of OP is the high morbidity of the procedure, specially due to the high blood transfusion rate $(7-14 \%)[3,4]$. HoLep is mainly limited by the steep learning curve, availability and postoperative transient urinary incontinence; however, it is superior to OP in terms of bleeding [5]. As well, BTUEP has a similar safety profile when compared to HoLep. Advantages of BTUEP include the widely available instrumentation and lower costs $[6,7]$. Finally, durability of endoscopic techniques over OP is still unclear.

The exact role of minimally invasive simple prostatectomy (MISP) is still unknown. MISP includes laparoscopic 
simple prostatectomy (LSP) and robot-assisted simple prostatectomy (RASP) $[8,9]$.The technique for LSP was first described in 2002 [10], while the first RASP was reported in 2008. According to the latest EAU guidelines, studies are needed to compare the efficacy, safety and hospitalization times of MISP and both OP and endoscopic procedures.

With this knowledge in mind, the aim of our study was to evaluate differences between BTUEP and LSP in terms of efficacy, quality of life (Qol) and perioperative complications.

\section{Methods}

A consecutive series of patients who underwent LSP in Pontevedra Hospital and BTUEP in Nuova Villa Claudia Hospital, between May 2012 and December 2015, were included in the study. Before surgery, all patients signed a dedicated informed consent and approved the use of their data for research purposes. Data were prospectively collected and an internal review board approved the retrospective analysis of the data set. All the procedures were performed in accordance with the Declaration of Helsinki. Any patient with prostate volume $(\mathrm{PV})<80 \mathrm{cc}$, incomplete data, a prior history of prostatic or urethral surgery, urethral stricture, neuro-vesical dysfunction and/or prostate cancer was excluded from the study. Pontevedra hospital performed only LSP procedures; while NVC clinic, only BTUEP procedures.

Patients with bothersome LUTS were offered surgery if preoperative IPSS $\geq 12$ points and/or QoL $\geq 4$ and/or maximal urinary flow rate $(\mathrm{Qmax})<15 \mathrm{~mL} / \mathrm{s}$ and/or post-void residual urine volume (PVR) $>50 \mathrm{~mL}$ and/or not-responding to medical therapy and/or not willing to undergo medical therapy [11].

Clinical data, including age, BMI, hemoglobin levels, International Prostate Symptom Score (IPSS), QoL score, prostate-specific antigen (PSA), PV and PVR, were collected.

All operations were performed by expert surgeons.

Primary endpoint of the study was to evaluate mediumterm efficacy in terms of symptoms, urinary flow and quality of life (Qol) of BTUEP vs LSP in patients with large prostates and lower urinary tract symptoms due to BPO. Secondary endpoints include safety and medium-term complications.

\section{Operative techniques}

\section{LSP}

LSP was exclusively performed in Pontevedra hospital. Patient lies in a modified supine position under general anesthesia. Trocars are positioned (Hasson over the umbilicus and then 1 of $12 \mathrm{~mm}$ and 3 of $5 \mathrm{~mm}$ ). A transversal incision is performed on the anterior wall of the prostate capsule for 3-4 $\mathrm{cm}$ to identify the prostate adenoma. We proceed with a blunt dissection in the avascular plane of the adenoma laterally and posteriorly. If a third lobe is identified, a dissection of the bladder mucosa is performed followed by a dissection of the posterior plane of the prostate to reach the apex. Finally, the adenoma is detached at the verumontanum level with cold scissors. Hemostasis is controlled using bipolar or monopolar electrocoagulation for minor vessels and two trans-capsular stiches for the pedicles. The trigonization of the prostatic fossa is performed using 2 or 3 stitches between the sacral lip of the bladder neck and posterior surgical capsule. A catheter is introduced. The prostatic capsule is reconstructed with running V-lock suture. The catheter is left inside for 5 days [12].

\section{BTUEP}

BTUEP was exclusively performed in Nuova Villa Claudia clinic. We performed an en bloc enucleation of the adenoma with an Olympus Surg-Master UES-40 bipolar generator, OES-Pro bipolar resectoscope, continuous-flow saline irrigation and 'button' type vapo-resection electrodes (Olympus Europe, Hamburg, Germany). Enucleation begins at the apex of the prostate just laterally to the verumontanum. The mucosa is vaporized and the plane between the adenoma and the capsule is identified. Thereafter, an incision is performed between the median and the left lobe until reaching the bladder neck at 5o'clock. The apical incision at 5 and 7 o'clock is extended with a latero-lateral movement and a disto-proximal compression of the surgeon on the button that creates progressive pedunculation plus tissue vaporization of the left adenoma. Coming back to the initial left apical incision, the mucosa is horizontally incised above the verumontanum, reaching the apex of the right lobe. After the incision of the mucosa within the cleft on the right side of the verumontanum, enucleation of the median and right lobes is carried out exactly as described for the left lobe from 5 to 9 o'clock, joining circumferentially its already detached superior part and the bladder neck. The en bloc enucleated adenoma is now completely isolated in one piece, but still fixed to the bladder neck. At this point, the pedunculated structures of the adenoma are resected with a loop. Meticulous coagulation of the prostatic loggia is then performed [13].

Intraoperative outcomes measured were operative time, weight of resected prostatic tissue and histological features. Perioperative outcomes were duration of bladder irrigation, time to catheter removal, hospital stay and postoperative hemoglobin levels (on day 1). Operative time in BTUEP patients was calculated as time between endoscope insertion to catheter positioning. Operative time in LSP was calculated as first trocar positioning to skin closure. 
During the follow-up patients underwent uroflowmetry, I.P.S.S. score, QoL score, PSA and PVR at 1, 3, 6, 12, 24 and 36 months. Both centers followed the patients with the same follow-up schedule.

Perioperative complications were graded according to Clavien Dindo classification as reported in previous experiences by our group [1]. Continence was defined as 'no-pads'.

Urethral stricture, bladder outlet obstruction (BOO), residual adenoma and postoperative acute urinary retention, as well as reintervention rate were also recorded.

\section{Statistical analysis}

Statistical analyses were performed using Statistical Package for the Social Sciences (SPSS Inc., Chicago, IL, USA). Continuous variables are presented as mean \pm standard deviation and were compared using the Mann- Whitney test. Categorical data (percentages) were compared using the chi-square test or Fisher's exact probability test. Differences from baseline were evaluated using the Wilcoxon test. $p$ values $<0.05$ were considered statistically significant. Post hoc power calculation was performed for primary outcomes (IPSS, Qmax and QoL) according to Levine et al. using an alpha value of 0,05 resulting in a power $>80 \%$ [14].

\section{Results}

Overall, 320 patients were evaluated and out of them, 296 patients presented complete data for the objectives of the study, 167/296 (54\%) underwent LSP and 129/296 (46\%) underwent BTUEP. At baseline patients undergoing BTUEP were older $(73,05 \pm 7.48$ vs $69,05 \pm 7,80 ; p=0,001)$ and presented lower prostate volumes $(94,80 \pm 14.99$ vs $108,1 \pm 38.98 ; p=0,001)$. Baseline characteristics and

Table 1 Baseline characteristics of both groups

\begin{tabular}{lccl}
\hline & LSP $(167 \mathrm{pts})$ & BTUEP $(129 \mathrm{pts})$ & $p$ \\
\hline Age (years) & $69,05 \pm 7,80$ & $73.05 \pm 7.48$ & 0.001 \\
$\mathrm{Hb}(\mathrm{g} / \mathrm{dl})$ & $14,4 \pm 1.41$ & $13.57 \pm 1.88$ & 0.225 \\
BMI $\left(\mathrm{kg} / \mathrm{m}^{2}\right)$ & $27.81 \pm 2.8$ & $28.09 \pm 2.63$ & 0.689 \\
I.P.S.S & $21,00 \pm 3,46$ & $23.15 \pm 5.65$ & 0.356 \\
Qmax (mL/s) & $9,53 \pm 4,2$ & $7.07 \pm 3,78$ & 0.568 \\
Qol & $2.1 \pm 0.53$ & $2.39 \pm 0.70$ & 0.856 \\
PVR $(\mathrm{mL})$ & $85 \pm 26.01$ & $85.29 \pm 26.13$ & 0.625 \\
TRUS $(\mathrm{cc})$ & $108,1 \pm 38.98$ & $94.80 \pm 14.99$ & 0.001 \\
PSA $(\mathrm{ng} / \mathrm{mL})$ & $7.86 \pm 5,6$ & $6.25 \pm 3.44$ & 0.253 \\
\hline
\end{tabular}

Data are presented as mean \pm standard deviation

IPSS International Prostate Symptom Score, BMI Body Mass Index, Qmax maximun flow rate, $P V R$ post-voided residual volume, IIEF International Index of Erectile Function, PSA prostate-specific antigen, $Q o l$ quality of life intraoperative and perioperative data are described in Tables 1 and 2. Overall, volume of tissue retrieved and postoperative $\mathrm{Hb}$ were similar in both groups $(p>0.05)$. The BTUEP procedure required significantly shorter operative time than the LSP procedure with a weighted mean difference (WMD) of - 33 min $(p<0.05)$ as well as shorter catheterization time with a WMD of -4.38 days $(p<0.001)$. Conversely, postoperative bladder irrigation time (WMD0.74 days, $p<0.001$ ) were significantly shorter in the LSP than in the BTUEP group.

Perioperative complications according to Clavien-Dindo are shown in Table 3. In terms of Grade I complications, patients undergoing BTUEP presented haematuria with clots removal more frequently when compared to LSP (7\% vs $2 \%, p<0,05)$. In terms of Grade II complications, there were no differences in terms of transfusion rates and AUR after catheter removal. As well, Grade III complications were comparable in both groups with reintervention rates ranging between 2 and 5\%. There were no grade IV and V complications.

Table 2 Peri and postoperative characteristics in both groups

\begin{tabular}{lrll}
\hline & \multicolumn{1}{l}{ LSP } & \multicolumn{1}{l}{ BTUEP } & \multicolumn{1}{l}{$p$} \\
\hline Surgery time (minutes) & $130,04 \pm 42,2$ & $97.02 \pm 25.90$ & 0.001 \\
$\begin{array}{l}\text { Volume of tissue retrieve } \\
\quad \text { grams) }\end{array}$ & $60.25 \pm 16.9$ & $59.36 \pm 17.45$ & 0.876 \\
$\begin{array}{l}\text { Percentage of prostate reduc- } \\
\quad \text { tion (\%) }\end{array}$ & $52,3 \pm 15,2$ & $59,5 \pm 19,3$ & 0.212 \\
Post-operative Hb (g/dl) & $12,34 \pm 6,75$ & $10.99 \pm 1.27$ & 0.275 \\
Bladder irrigation (days) & $0,93 \pm 0,47$ & $1,67 \pm 0,63$ & 0.001 \\
Catheterization time (days) & $6,9 \pm 5,15$ & $2,52 \pm 0,67$ & 0.001 \\
Hospital Stay (days) & $3,3 \pm 0,66$ & $3,21 \pm 0,63$ & 0.821 \\
\hline
\end{tabular}

Data are presented as mean \pm standard deviation

Table 3 Complications according to the Clavien classification system

\begin{tabular}{llll}
\hline Complications & LSP (167pts) & BTUEP (129 pts) & $p$ \\
\hline Grade I & & & \\
Transient Hematuria & $12(7,1)$ & $2(1,55)$ & 0.021 \\
UTI & $4(2)$ & $5(4)$ & 0,249 \\
Acute urinary retention & $11(6,5)$ & $5(4,1)$ & 0.301 \\
$\quad$ Transient urinary incon- & $3(2)$ & $2(1,5)$ & 0,778 \\
$\quad$ tinence & & & \\
$\quad$ Total & $30(18)$ & $14(11)$ & 0,133 \\
Grade II & & & \\
$\quad$ Transfusions & $15(9)$ & $12(9)$ & 0.898 \\
Grade III & & & \\
$\quad$ Reintervention $(\%)$ & $3(1,7)$ & $6(4,65)$ & 0.156 \\
Grade IV & 0 & 0 & \\
Grade V & 0 & 0 & \\
\hline
\end{tabular}


Table 4 describes functional outcomes at 1, 3, 6, 12, 24 and 36 months. At each time point, the LSP group presented statistically significant improvements when compared to baseline in urinary symptoms (Range of mean IPSS improvement: 17,3-20,6), quality of life (Range of mean QoL improvement: 2-3), urinary flow (Range of mean Qmax

Table 4 Functional outcomes after LSP and BTUEP

\begin{tabular}{|c|c|c|c|}
\hline & LSP group & BTUEP group & $p$ \\
\hline \multicolumn{4}{|l|}{ IPSS } \\
\hline Baseline & $21,00 \pm 3,46$ & $23.15 \pm 5.65$ & 0.356 \\
\hline 1 month & $5.5 \pm 4.4$ & $5.8 \pm 3.41$ & 0.882 \\
\hline 3 months & $4.2 \pm 3.16$ & $4.35 \pm 3.1$ & 0.326 \\
\hline 6 months & $3.03 \pm 2.56$ & $3.06 \pm 1.29$ & 0.453 \\
\hline 12 months & $3.02 \pm 4.14$ & $2.68 \pm 0.98$ & 0.523 \\
\hline 24 months & $2,90 \pm 0.62$ & $2.66 \pm 1.01$ & 0.325 \\
\hline 36 months & $2,56 \pm 1.43$ & $2.62 \pm 0.78$ & 0.423 \\
\hline \multicolumn{4}{|l|}{ Qol } \\
\hline Baseline & $2.1 \pm 0.53$ & $2.39 \pm 0.70$ & 0.856 \\
\hline 1 month & $5.13 \pm 1.45$ & $4.02 \pm 1.11$ & 0.856 \\
\hline 3 months & $5.14 \pm 1.55$ & $4.23 \pm 1.15$ & 0.754 \\
\hline 6 months & $4.26 \pm 0.91$ & $5.06 \pm 0.86$ & 0.956 \\
\hline 12 months & $4.44 \pm 0.84$ & $5.25 \pm 0.63$ & 0.965 \\
\hline 24 months & $4.50 \pm 0.82$ & $5.10 \pm 0.67$ & 0.754 \\
\hline 36 months & $4.36 \pm 0.73$ & $5.26 \pm 0.63$ & 0.954 \\
\hline \multicolumn{4}{|l|}{ Qmax } \\
\hline Baseline & $9,53 \pm 4,2$ & $7.07 \pm 3,78$ & 0.568 \\
\hline 1 month & $17.4 \pm 10.25$ & $17.41 \pm 5.75$ & 0.854 \\
\hline 3 months & $24,8 \pm 10,7$ & $20.7 \pm 7.36$ & 0.656 \\
\hline 6 months & $25.72 \pm 9.56$ & $21.7 \pm 7.22$ & 0.864 \\
\hline 12 months & $22.62 \pm 8.82$ & $23.14 \pm 7.25$ & 0.532 \\
\hline 24 months & $22.73 \pm 8,98$ & $22.18 \pm 7.13$ & 0.678 \\
\hline 36 months & $22.21 \pm 7.39$ & $22.88 \pm 7.04$ & 0.436 \\
\hline \multicolumn{4}{|l|}{ PVR } \\
\hline Baseline & $85 \pm 26.01$ & $85.29 \pm 26.13$ & 0.625 \\
\hline 1 month & $18.8 \pm 17.86$ & $35.2 \pm 23.86$ & 0.754 \\
\hline 3 months & $38.2 \pm 15.02$ & $28.2 \pm 17.06$ & 0.954 \\
\hline 6 months & $32.9 \pm 13.17$ & $17.75 \pm 9.86$ & 0.365 \\
\hline 12 months & $33.24 \pm 13.26$ & $18.6 \pm 9.86$ & 0.105 \\
\hline 24 months & $32.69 \pm 12.2$ & $18.8 \pm 9.86$ & 0.111 \\
\hline 36 months & $35.69 \pm 11.5$ & $17.8 \pm 8.28$ & 0.062 \\
\hline \multicolumn{4}{|l|}{ PSA } \\
\hline Baseline & $7.86 \pm 5,6$ & $6.25 \pm 3.44$ & 0.253 \\
\hline 6 months & $2,15 \pm 0.81$ & $2.38 \pm 0.74$ & 0.656 \\
\hline 12 months & $2,20 \pm 0.72$ & $2.39 \pm 0.76$ & 0.402 \\
\hline 24 months & $2,07 \pm 0.62$ & $2.56 \pm 0.56$ & 0.545 \\
\hline 36 months & $2,22 \pm 0.61$ & $2.37 \pm 0.73$ & 0.312 \\
\hline
\end{tabular}

Data are presented as mean \pm standard deviation

IPSS International Prostate Symptom Score, BMI Body Mass Index, Qmax maximun flow rate, $P V R$ post-voided residual volume, $P S A$ prostate-specific antigen, $Q o l$ quality of life, BTUEP bipolar transurethral enucleation of the prostate improvement: 10-16 mL/s), PSA levels (Range of mean PSA reduction: 3,6-3,8) and residual urine ( Range of mean PVR reduction: 49-66 cc). As well at each time point, the BTUEP group presented statistically significant improvements when compared to baseline in urinary symptoms ( Range of mean IPSS improvement: 15,5-18,1), quality of life (Range of mean QoL improvement: 2-3), urinary flow (Range of mean Qmax improvement: 7-15 mL/s), PSA levels (Range of mean PSA reduction: 5,6-5,7) and residual urine ( Range of mean PVR reduction: 49-66 cc),

As well, at each time point (1, 3, 6, 12, 24 and 36 months), there were no statistically significant differences between groups in terms of IPSS, QoL. Qmax, PSA values and PVR improvements $(p>0,05)$ (Table 4).

Overall, 250/296 (85\%) patients completed the 3-year follow-up period (Table 5). During this period, 20 patients were diagnosed of cancer, 20 patients needed a reintervention and 6 patients died of non-urological conditions. Reintervention rate was $4 \%$ in the LSP group (4 cases of bladder-neck contracture and 3 urethral strictures) and 5\% in BTUEP group (4 bladder-neck contracture and 2 urethral strictures). All the reinterventions occurred in the first year of follow-up.

\section{Discussion}

The present study compares for the first time BTUEP and LSP for the treatment of large adenomas in patients with BPO. According to our results, both techniques are to be considered equal in terms of functional outcomes. LSP showed longer operative times and longer catheterization times when compared to BTUEP. However, irrigation time was shorter in the LSP group. Both techniques presented good safety profiles with similar transfusion and reintervention rates. Our results are in line with the peer-reviewed literature $[15,16]$.

The goal of prostate surgery for BPH is to remove the adenoma while minimizing the damage to surrounding structures. According to the latest EAU guidelines, in prostates larger than $80 \mathrm{cc}$, surgeons should choose between OP, BTUEP or HoLEP [2].

Table 5 Number of patients available for analysis

\begin{tabular}{lll}
\hline & BTUEP group & LSP group \\
\hline Baseline & 129 & 167 \\
1 month & 129 & 167 \\
3 months & 123 & 159 \\
6 months & 120 & 157 \\
12 months & 115 & 150 \\
24 months & 107 & 147 \\
36 months & 105 & 145 \\
\hline
\end{tabular}


In the past years, LSP has been evaluated by several authors. According to our results, LSP improves symptoms and quality of life at three years (mean IPSS scores: $2,5 \pm 1,4$; mean Qol score of 4,4 $\pm 0,7$ ). As well, we observed significant improvements in Qmax and PVR (mean Qmax: $21 \pm 7.39 \mathrm{~mL} / \mathrm{s}$; mean PVR of $35.69 \pm 11.5 \mathrm{~mL}$ ). Our results are in line with the largest multi-center outcome analysis of the minimally invasive approaches to simple prostatectomy by Autorino et al. The study evaluated 1330 MISP between 2000 and 2014. Of these, 487 (36.6\%) were robotic and 843 $(63.4 \%)$ were pure laparoscopic. They observed significant improvement in functional outcomes at 12-month followup $[12,17]$. Post-operative median Qmax and median IPSS were 22 (IQR: 20-26) mL/s and 5 (IQR:3-5), respectively, in the laparoscopic group versus 25 (IQR:20-33) $\mathrm{mL} / \mathrm{s}$ and 7 (IQR:4-9), respectively, in the robotic group.

In our study, we evaluated two minimally invasive techniques. The concept of minimally invasive is still a matter of debate in the literature. Laparoscopy may be considered minimally invasive specially if compared with open prostatectomy; however, it still requires general anesthesia, a Trendelenburg position as well as long catheterization time [13]. It is important to underline that the differences in catheterization time clearly depend on surgical protocol. More specifically, patients on the LSP group routinely remove catheter on day 5, while patients on the BTUEP group on day 2. Probably endoscopic procedures are less invasive when compared to laparoscopy, considering that patient has no need of general anesthesia and has shorter catheterization time. However, in very large prostates (>200 cc), endoscopy may be challenging and time consuming [18]. In our experience, both techniques showed good functional outcomes and safety profiles at three years. Standing to these results both techniques may be proposed to the patient, which should be aware of the pros and cons of each procedure to make an informed decision [19].

We observed a good safety profile for both techniques; however, some difference were recorded. BTUEP showed higher rates of clots removal when compared to LSP, which has the advantage of a direct coagulation of the whole prostatic loggia. Interestingly, LSP and BTUEP presented similar transfusion rates $(9 \%)$ and reintervention rates $(2-5 \%)$. In the BTUEP group, transfusion rate was higher than reported in the literature. A possible explanation for this difference is that our series include old patients with low preoperative $\mathrm{Hb}$ levels as well as patients with prostates larger than 140 cc. Conversely, the transfusion rate in the LSP group is slightly lower than the available literature [20]. In our cohort patients, some patients needed reintervention (4-6\%). All these patients were evaluated initially with a TRUS to diagnose residual adenoma, with cystoscopy to diagnose urethral stricture and with an urodynamic investigation to confirm obstruction. In both groups, all patients were managed endoscopically. The results are in line with the available evidence on HoLEP [21] on bipolar electrosurgical enucleation of the prostate (BEEP) and on MISP [22, 23].

Secondary endpoints of the study include operative time, length of hospitalization and catheterization period. According to our results, BTUEP showed a more favorable profile compared to LSP considering the mean operative times $(130,04 \pm 42,2 \mathrm{~min}$ vs $97.02 \pm 25.90 \mathrm{~min}, p=0,001)$ and mean catheterization period $(6,9 \pm 5,1$ vs $2,52 \pm 0,67$, $p=0,001)$. The longer catheterization depends on the different operative technique considering that LSP requires to open the prostatic capsule with subsequent suturing; however, most of the patients may go home on day $2 / 3$ with the catheter considering that by that time urine is usually clear. Our results are in line with the available evidence comparing these two procedures [24-26].

Although not evaluated in this study, technical aspects, learning curve and costs are important issues when choosing the best surgical option. BTUEP and LSP can be considered easy techniques for surgeons already trained.

In terms of costs, considering that hospital stay is similar, it is assumable that LSP is more expensive than BTUEP in terms of material costs; however, dedicated studies should assess this issue.

Although some differences between different procedures exist, all of them seem equally effective in treating large-volume adenomas. In our study, we have the merit of comparing two techniques performed in highly experienced centers. In each center, a consecutive series of patients undergoing BTUEP (Nuova Villa Claudia) and LSP (Pontevedra Hospital) were enrolled. Although this could be considered a possible limitation, both techniques are considered challenging procedures and so far we preferred to plan the study in centers with a long-lasting experience with these techniques. Our results clearly depend on our population and on patient selection and our results cannot be extended to other centers with no or minimal experience with these techniques. However, the present study is the only available evidence comparing these two techniques in highly experienced centers.

This study presents some limitations. First, the retrospective fashion of the study is a major limitation; however, to overcome this limitation, both databases are prospectively maintained database which were thereafter analyzed retrospectively.

Another important limitation of our study is the fact that all the surgeries were performed by highly experienced endoscopic and laparoscopic surgeons and therefore, the results cannot be extended to all centers. Moreover, our results clearly depend on the enrolled population and cannot be extended to other populations. The lack of urodynamic studies may be considered another limitation. However, most of the studies on endoscopic surgery do not include urodynamic studies [27]. Furthermore, according to latest EAU 
guidelines, the use of invasive urodynamics studies should be considered only in selected patients which were not included in our study. As well the lack of data on pain management and time to work may be considered a limitation.

We agree that more than one study is necessary to prove a hypothesis. Notwithstanding all these limitations, this study is the only available study comparing LSP and BTUEP outcomes and safety at three years.

\section{Conclusion}

LSP showed similar outcomes and safety at three years when compared to BTUEP in terms of symptoms, urinary flow, Qol, PVR and PSA. However, LSP presented a longer operative, bladder irrigation and catheterization time. Our study opens new insights on the role of BTUEP and LSP for the management of large prostates in patients with LUTS due to BPH. Further studies should clarify the role of these techniques, include cost analysis evaluation and define the best patient profile for both procedures. Theoretically, a tertiary referral center and a BPH clinic should include several surgical options including BTUEP and LSP to tailor the best procedure according to the patient's characteristics and expectations.

\section{Acknowledgements None.}

Author contributions RL: conceived and designed the work that led to the submission. Drafted revised the manuscript and approved the final version. GT: conceived the work that led to the submission, acquired data, revised the manuscript. Approved the final version. AZA: acquired data, played an important role in interpreting the results, revised the manuscript. Approved the final version. CPA: Acquired data, played an important role in interpreting the results, revised the manuscript. Approved the final version. ABM: conceived and designed the work that led to the submission. Drafted revised the manuscript and approved the final version. HRN: acquired data, played an important role in interpreting the results, revised the manuscript. Approved the final version. JAGD: conceived the work that led to the submission, acquired data, revised the manuscript. Approved the final version. BCG Conceived the work that led to the submission, acquired data, revised the manuscript. Approved the final version. LA: conceived the work that led to the submission, acquired data, revised the manuscript. Approved the final version. LM: conceived the work that led to the submission, acquired data, revised the manuscript. Approved the final version. FC: conceived the work that led to the submission, acquired data, revised the manuscript. Approved the final version. VB: conceived the work that led to the submission, acquired data, revised the manuscript. Approved the final version. AT acquired data, played an important role in interpreting the results, revised the manuscript. Approved the final version. CDN: conceived the work that led to the submission, acquired data, revised the manuscript. Approved the final version. MRM: conceived and designed the work that led to the submission. Drafted revised the manuscript and approved the final version. RG: conceived and designed the work that led to the submission. Drafted revised the manuscript and approved the final version.
Funding Open access funding provided by Università degli Studi di Roma La Sapienza within the CRUI-CARE Agreement.

\section{Compliance with ethical standards}

Conflict of interest The authors have declared no conflicts of interest.

Ethical approval This research involves Human Participants, it does not involve animals and every patient enrolled have signed an informed consent.

Open Access This article is licensed under a Creative Commons Attribution 4.0 International License, which permits use, sharing, adaptation, distribution and reproduction in any medium or format, as long as you give appropriate credit to the original author(s) and the source, provide a link to the Creative Commons licence, and indicate if changes were made. The images or other third party material in this article are included in the article's Creative Commons licence, unless indicated otherwise in a credit line to the material. If material is not included in the article's Creative Commons licence and your intended use is not permitted by statutory regulation or exceeds the permitted use, you will need to obtain permission directly from the copyright holder. To view a copy of this licence, visit http://creativecommons.org/licenses/by/4.0/.

\section{References}

1. De Nunzio C, Lombardo R, Autorino R et al (2013) Contemporary monopolar and bipolar transurethral resection of the prostate: prospective assessment of complications using the Clavien system. Int Urol Nephrol 45:951-959

2. Gratzke C, Bachmann A, Descazeaud A et al (2015) EAU guidelines on the assessment of non-neurogenic male lower urinary tract symptoms including benign prostatic obstruction. Eur Urol 67:1099-1109

3. Kuntz RM, Lehrich K, Ahyai SA (2008) Holmium laser enucleation of the prostate versus open prostatectomy for prostates greater than 100 grams: 5-year follow-up results of a randomised clinical trial. Eur Urol 53:160-168

4. Varkarakis I, Kyriakakis Z, Delis A et al (2004) Long-term results of open transvesical prostatectomy from a contemporary series of patients. Urology 64:306-310

5. Sivarajan G, Borofsky MS, Shah O et al (2015) The role of minimally invasive surgical techniques in the management of largegland benign prostatic hypertrophy. Rev Urol 17:140-149

6. Giulianelli R, Gentile BC, Mirabile G et al (2017) Bipolar plasma enucleation of the prostate (B-TUEP) in benign prostate hypertrophy treatment: 3-year results. Urology 107:190-195

7. Giulianelli R, Gentile B, Albanesi L et al (2015) Bipolar button transurethral enucleation of prostate in benign prostate hypertrophy treatment: a new surgical technique. Urology 86:407-413

8. Leonardo C, Lombardo R, Cindolo L et al (2019) What is the standard surgical approach to large volume BPE? Systematic review of existing randomized clinical trials. Minerva Urol Nefrol 72:22-29

9. Shah AA, Gahan JC, Sorokin I (2018) Comparison of robotassisted versus open simple prostatectomy for benign prostatic hyperplasia. Curr Urol Rep 19:71

10. Mariano MB, Graziottin TM, Tefilli MV (2002) Laparoscopic prostatectomy with vascular control for benign prostatic hyperplasia. J Urol 167:2528-2529 
11. Asimakopoulos AD, De Nunzio C, Kocjancic E et al (2016) Measurement of post-void residual urine. Neurourol Urodyn 35:55-57

12. Autorino R, Zargar H, Mariano MB et al (2015) Perioperative outcomes of robotic and laparoscopic simple prostatectomy: a European-American Multi-institutional Analysis. Eur Urol 68:86-94

13. Giulianelli R, Gentile BC, Mirabile G et al (2019) Bipolar plasma enucleation of the prostate vs open prostatectomy in large benign prostatic hyperplasia: a single centre 3-year comparison. Prostate Cancer Prostatic Dis. 22:110-116

14. Levine M, Ensom MHH (2001) Post hoc power analysis: an idea whose time has passed? Pharmacotherapy 21:405-409

15. Pirozzi L, Sountoulides P, Castellan P et al (2015) Current pharmacological treatment for male LUTS due to BPH: Dutasteride or Finasteride? Curr Drug Targets 11:1198-1206

16. De Nunzio C, Lombardo R, Gacci M et al (2015) The diagnosis of benign prostatic obstruction: validation of the young academic urologist clinical nomogram. Urology 86:1032-1036

17. Pariser JJ, Packiam VT, Adamsky MA et al (2016) Trends in simple prostatectomy for benign prostatic hyperplasia. Curr Urol Rep 17:57

18. Nestler S, Bach T, Herrmann T et al (2019) Surgical treatment of large volume prostates: a matched pair analysis comparing the open, endoscopic (ThuVEP) and robotic approach. World J Urol 37:1927-1931

19. De Nunzio C, Presicce F, Lombardo R et al (2018) Patient centred care for the medical treatment of lower urinary tract symptoms in patients with benign prostatic obstruction: a key point to improve patients' care-a systematic review. BMC Urol 18:62

20. Lucca I, Shariat SF, Hofbauer SL et al (2015) Outcomes of minimally invasive simple prostatectomy for benign prostatic hyperplasia: a systematic review and meta-analysis. World J Urol 33:563-570
21. Naspro R, Suardi N, Salonia A et al (2006) Holmium laser enucleation of the prostate versus open prostatectomy for prostates $>70 \mathrm{~g}$ : 24-month follow-up. Eur Urol 50:563-568

22. Rao J-M, Yang J-R, Ren Y-X et al (2013) Plasmakinetic enucleation of the prostate versus transvesical open prostatectomy for benign prostatic hyperplasia $>80 \mathrm{~mL}$ : 12 -month follow-up results of a randomized clinical trial. Urology 82:176-181

23. Pavan N, Zargar H, Sanchez-Salas R et al (2016) Robot-assisted versus standard laparoscopy for simple prostatectomy: multicenter comparative outcomes. Urology 91:104-110

24. Geavlete B, Stanescu F, Iacoboaie C et al (2013) Bipolar plasma enucleation of the prostate vs open prostatectomy in large benign prostatic hyperplasia cases-a medium term, prospective, randomized comparison. BJU Int 111:793-803

25. Elzayat EA, Elhilali MM (2006) Holmium laser enucleation of the prostate (HoLEP): the endourologic alternative to open prostatectomy. Eur Urol 49:87-91

26. Vavassori I, Hurle R, Vismara A et al (2004) Holmium laser enucleation of the prostate combined with mechanical morcellation: two years of experience with 196 patients. J Endourol 18:109-112

27. Cornu JN, Ahyai S, Bachmann A et al (2015) A systematic review and meta-analysis of functional outcomes and complications following transurethral procedures for lower urinary tract symptoms resulting from benign prostatic obstruction: an update. Eur Urol 67:1066-1096

Publisher's Note Springer Nature remains neutral with regard to jurisdictional claims in published maps and institutional affiliations.

\section{Affiliations}

\section{Riccardo Lombardo ${ }^{1}$ - Anton Zarraonandia Andraca ${ }^{2}$. Cristina Plaza Alonso ${ }^{2}$. Juan Andres González-Dacal ${ }^{2}$. Higinio Rodríguez Núñez ${ }^{2}$. Aaron Barreiro Mallo ${ }^{2}$. Barbara Cristina Gentile ${ }^{1}$ Giorgia Tema ${ }^{1}$. Luca Albanesi ${ }^{1}$.

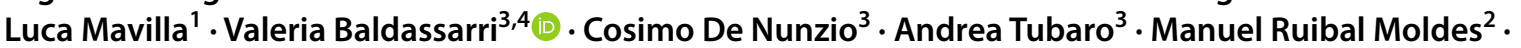 Roberto Giulianelli'}

Riccardo Lombardo

rlombardo@me.com

Anton Zarraonandia Andraca

andraca.az@gmail.com

Cristina Plaza Alonso

cpalonso@yahoo.sp

Juan Andres González-Dacal gonzales.juan@gmail.sp

Higinio Rodríguez Núñez nunezhiginio@gmail.com

Aaron Barreiro Mallo aaronbmallo@yahoo.sp

Barbara Cristina Gentile gentilebarbara@hotmail.com

Giorgia Tema

giorgiat88@hotmail.it

Luca Albanesi

albanesiluca@yahoo.it
Luca Mavilla

1.mavilla@gmail.it

Cosimo De Nunzio

cosimodenunzio@libero.it

Andrea Tubaro

andrea.tubaro@uniroma1.it

Manuel Ruibal Moldes

moldes-mr@yahoo.sp

Roberto Giulianelli

rgiulianelli@gmail.com

1 Nuova Villa Claudia, Rome, Italy

2 Complejo Hospitalario de Pontevedra Hospital Montecelo, Pontevedra, Spain

3 Azienda Ospedaliera Sant'Andrea Roma, 'Sapienza' University, Rome, Italy

4 Department of Urology, University "La Sapienza", Rome, Italy 